made the development in this part parallel to but independent of those in Chapter III. This brings out very clearly the analogy and differences between the problems of one-dimensional waves and those of steady plane flows. Besides such topics as the hodograph transformation, limiting lines, simple waves, there is an extensive discussion of shock interaction and reflection. The last part of this chapter deals with the exact nature of problems of steady flow past an obstacle, including an extensive and systematic discussion of the perturbation theory. This part should be highly recommended to the inquisitive workers in this field.

Two more short chapters follow. Chapter $\mathrm{V}$ deals with flow in nozzles and jets. Chapter VI deals with three-dimensional flows having suitable symmetry properties, so that there are still two independent variables. The book ends with an extensive and valuable list of references to books and other publications.

In conclusion, the reviewer wishes to recommend this book to every worker in the field of dynamics of a compressible fluid, whether he is studying it from the point of view of a mathematician, a physicist, or an engineer.

C. C. LIN

Moderne algebraische Geometrie. Die idealtheoretischen Grundlagen. By W. Gröbner. Vienna, Springer, 1949. $12+212$ pp. $\$ 5.70$.

Dr. Gröbner's book is a textbook giving the fundamentals of the ideal theory needed in algebraic geometry. The exposition is clear, elegant, and easy to read.

About one-half of the material-basic field theory, the ideal theory of polynomial rings, and the more general commutative ideal theory - can be found in van der Waerden's textbook on algebra; the remainder-material on Hilbert's function, the ring of formal power series, integral algebraic quantities, and the syzygy theory of homogeneous polynomial ideals - can be found without too much difficulty in the literature, but appears here for the first time in textbook form.

Dr. Gröbner has organized all this material excellently, occasionally improving known proofs-especially in the resultant theory and the syzygy theory - and has at all times kept the geometry in the foreground; the motivation of the development of each subject is thus at all times quite clear.

As is obvious, different ideals in a polynomial ring can have the same locus of zeros-in Dr. Gröbner's terminology, the same "Nullstellengebilde" (NG). The author attempts to establish a 1-1 correspondence between ideals and geometric objects, associating with 
every polynomial ideal its "algebraische Mannigfaltigkeit" (AM), a term used here in a sense different from the customary. The AM of an ideal is to consist, namely, of its NG together with certain "infinitely near" loci. But the author does not go beyond a few suggestive remarks, and the main work on the idea remains to be carried out, if it can be at all.

The chapter on syzygy theory is an original contribution of Dr. Gröbner, and has appeared in the Monatshefte für Mathematik vol. 53 (1949) pp. 1-16. A part of this paper has been criticized by $P$. Dubreil in the Comptes Rendus, Académie des Sciences, Paris vol. 229 (1949) pp. 11-12, and the criticism applies also to some extent to the book. (Dubreil's and Gröbner's papers are reviewed in Mathematical Reviews vol. 11 (1950) p. 489.)

While illustrative examples are occasionally given in the footnotes, Dr. Gröbner could increase the usefulness of his textbook by including exercises and also further references to the literature.

\section{A. SEIDENBERG}

The variational principles of mechanics. By Cornelius Lanczos.

(Mathematical Expositions, no. 4.) Toronto, University of Toronto Press, $1949.25+307$ pp. $\$ 5.75$.

The most outstanding feature of this book is the enthusiastic style in which it is written. The enthusiasm is contagious to the extent that even the most iconoclastic reader can not but be intrigued by "Lagrange's ingenious idea" (on p. 39), by d'Alembert's stroke of genius (p. 88), by Gauss's "ingenious reinterpretation" thereof ( $p$. 106), by the "amazing result" of Hamilton (p. 220), or by the biblical quotation at the head of the eighth Chapter: "Put off thy shoes from off thy feet, for the place whereon thou standest is holy ground."

The author gives on the whole an able exposition of the following topics: The Euler-Lagrange equations of the calculus of variations, d'Alembert's principle, the principle of least constraint, the Lagrangian equations of motion, principle of Hamilton, principle of least action, integration by ignoration of coordinates, the Legendre's transformation, the canonical equations of motion, integral invariants, canonical transformations, the brackets of Lagrange and Poisson, infinitesimal canonical transformations, the partial differential equation of Hamilton and Jacobi, solution by separation of variables, Delaunay's treatment of separable periodic systems, the significance of all this material in the development of both the older and more recent quantum mechanics.

In addition to the final chapter devoted to a brief historical survey, 